\title{
Intrathecal Mesenchymal Stem Cell Therapy in Multiple Sclerosis: A Follow- Up Study for Five Years After Injection
}

\author{
Mohammad Ali Sahraian ${ }^{1,2,}$, Mandana Mohyeddin Bonab ${ }^{3}$, Sanaz Ahmadi Karvigh ${ }^{1}$, \\ Sepideh Yazdanbakhsh ${ }^{4}$, Behrouz Nikbin ${ }^{3}$, Jamshid Lotfi ${ }^{4}$ \\ ${ }_{2}^{1}$ MS Research Center, Neuroscience Institute, Tehran University of Medical Sciences, Tehran, IR Iran \\ ${ }_{3}^{2}$ Department of Neurology, Sina Hospital, Tehran University of Medical Sciences, Tehran, IR Iran \\ ${ }^{3}$ Department of Immunology, Tehran University of Medical Sciences, Tehran, IR Iran \\ 4 Iranian MS Society, Tehran, IR Iran \\ ${ }^{*}$ Corresponding author: Mohammad Ali Sahraian, Department of Neurology, Sina Hospital, Hasan Abad Sq., Tehran, IR Iran. Tel.: +98-2166706142, Fax: +98-2166706141, E-mail: msah- \\ rai@sina.tums.ac.ir.
}

Received: July 19, 2013; Revised: September 27, 2013; Accepted: September 28, 2013

\begin{abstract}
Background: Mesenchymal stem cell therapy has been used in multiple sclerosis (MS) in order to modulate the course of the disease in previous studies. One of the major concerns in such cases is long term safety or efficacy of this type of therapy.

Objectives: This study was conducted to report the clinical status of five patients with secondary progressive multiple sclerosis and one patient with neuromyelitis optica, five years after an autologous intrathecal mesenchymal stem cell (MSC)injection.

Patients and Methods: The patients (three male, three female) had a progressive course nonresponsive to the conventional immunomodulatory treatments with Expanded Disability Status Scale (EDSS) score of 3.5 to 6. They received the MCSs after discontinuing other treatments. They were examined annually to assess the disease activity and possible complications.

Results: Two patients had no change in their EDSS scores. One was diagnosed to have Devic's disease decreased one score in the EDSS, but experienced four relapses during these five years. Three patients had an increase in EDSS scores by 1-2 scores after five years. Two experienced relapses after injection. There was no significant adverse reaction, infection, or neoplasm during this period of follow up. Conclusions: Intrathecal mesenchymal stem cell therapy for MS is generally safe and did notresult in any adverse reaction like malignancy for a relatively long period of time. At least half of the patients had no change in their EDSS and the remaining patients had a delay in disease progress.
\end{abstract}

Keywords: Multiple Sclerosis; Stem Cells; Mesenchymal Stem Cell Transplantation; Tissue Therapy

\section{Background}

Multiple sclerosis (MS) is a chronic demyelinating disease of the central nervous system that usually affects young adults. It leads to substantial disability through deficits of motor, sensation, and neurocognitive functions. About $85 \%$ of cases have a relapsingremitting (RR) course at the beginning, which moves through periodic exacerbations with subsequent full or partial recovery before entering a progressive form. Unfortunately, around $65 \%$ - \%80 of patients enter this secondary progressive phase, in which any recovery of function is rare. After 15 years of disease, 50\% of patients become dependent on at least a walking aid (1, 2 ). Despite major progress in our understanding of the immunopathology of this disease over the past thirty years and development of immunomodulatory and immunosuppressive therapies for MS, there is no definite cure for this disabling disorder. The currently available therapies for MS are either partially effective or may cause serious adverse events after long-term application (3). In the past decade, stem cell transplantation has shown promises as an alternative immunoregulatory treatment in many inflammatory diseases, including MS $(3,4)$. Stem cell therapy also has the advantage of assisting the repair and remyelination process compared to other available treatments (5-7). Different stem cell types (neural derived, mesenchymal, embryonic), sources (allogenic, autologous), and various roots of administration (intravenous, intratechal, mixed) have been proposed for transplantion. Mesenchymal stem cells (MSCs) are a rare, heteroge- 
neous conglomerate of cells, which can be isolated from most connective tissues in the body and have the ability to differentiate into bone, fat, cartilage, and to a lesser extent into neurectoderm $(6,8)$. Bone marrowmesenchymal stem cell (BM-MSC) can promote neuroprotection by inhibiting gliosis, scar formation, and apoptosis, and by stimulating local progenitor cells (9). Injection of human or murine BM-MSCs in mouse with experimental allergic encephalomyelitis (animal model of MS) resulted in improved clinical outcomes (10-14). Clinical trials with MSCs on patients with MS have started during recent years (15-17). Most of these reports have indicated the safety and relative efficacy of stem cell therapy in MS (18). Although, there are few studies that convey concerns about the possible long term adverse effects $(6,14)$, numerous preliminary reports and ongoing trials are focused on this issue.

\section{Objectives}

Here, we report an extended follow up on six patients with MS who were treated with autologous intrathecal mesenchymal stem cell (MSC) five years ago, and their initial report after a one-year follow up was published in 2007 (16). Actually our previous study was the first published data on application of intrathecal mesenchymal stem cell in MS, and this long term follow up of the patients can shed more lights on the safety and efficacy of this method.

\section{Patients and Methods}

\subsection{Patients}

We originally enrolled 10 MS patients (secondary progressive) who, despite the routine immunomodulatory treatments (interferons and mithoxantrone), were non responsive with progressing disability. Unfortunately we lost track of 4 of our patients because they refused to participate in the research process. Of the remaining 6 that we could follow, 3 were females. The mean age of the patients was $28 \pm 4.3$ years. At the time of enrolment, all of them were diagnosed with a secondary progressive (SP) MS, although, one of our patients was later diagnosed to have Devic's disease. The inclusion criteria for injection included; an Expanded Disability Status Scale (EDSS) score of less than 6, increased EDSS scores (at least 1 score) in the previous year before injection, lack of a serious concomitant disease, failure to response to the other available treatments (Betainterferons and Mitoxantrone), and age of under 60 years. The treatment protocol was approved by the ethics committee of Tehran University of Medical Sciences (FWA00001331) and all subjects provided signed informed consent forms.

\subsection{Methods}

\subsubsection{Sample Collection and Mesenchymal Stem Cell Expansion}

Autologous mesenchymal stem cells were used for this study. The samples were obtained through $40 \mathrm{~mL}$ bone marrow (BM) aspiration two months prior to injection. Then the BM mononuclear cells (MNCs) were separated by the Ficoll density gradient method. Then they were incubated at $37{ }^{\circ} \mathrm{C}$ in a humidified atmosphere containing 5\% $\mathrm{CO}_{2}$ and were nourished with complete medium replacement every four days, until the fibroblast-like cells, at the base of the flask, reached confluence. The adherent cells were re-suspended, reseeded, and incubated again. Passage was repeated until a required number of cells was achieved. By the end of final passage, they were washed and supplemented with $1 \%$ human serum albumin and heparin, then they were re-suspended at a density of 1 $1.5 \times 106$ cells/mL density (16). Immunophenotyping was performed via typical flow cytometry profile and immunocytochemistry staining to isolate MSCs (CD13/CD44 and CD105/CD166 positive but CD45/CD34 and CD31 negative). The viability of the cells was confirmed just before injection. Bacteriological tests were performed after each passage and at the time of injection to ensure the safety of the samples. The thorough details of the process of preparing the MSCs are available in our former report (16).

\subsubsection{Injection of Mesenchymal Stem Cells}

A mean volume of $5.5 \mathrm{~mL}$ was injected intrathecally. The injected MSC count ranged between $(2.5-21) \times 106$ $($ mean $=7.5 \times 106)$. The patients were carefully observed for any adverse reactions for the next 24 hours. They were visited by a neurologist every month at first year and twice a year for the remaining four years. The patients were not given any conditioning regimen or immunosuppressive drugs immediately before or after MSC injection for one year, then each continued on a conventional disease modifying therapy according to their personal physicians' advice. The results of the first two years were published previously and the authors followed the patients for the next three years to see if there is any new complication or major adverse event related to this type of cell therapy.

\subsubsection{Neuroimaging Protocol}

All study subjects underwent brain MRI at the study entry, and after 12 months, using a $1.5 \mathrm{~T}$ machine with a standard quadrature head coil. The imaging protocol consisted of sagittal T1-, axial T1, T2-weighted images, and fluid attenuated inversion recovery (FLAIR) images. All MRI scans were performed before and after infusion 
of Gd-DTPA. We used axial T2-weigthed and pre- and post-contrast T1-weighted images for assessment of MS lesions. The images were independently interpreted using inspection and computer-assisted techniques by a neuroradiologist blinded to subjects' clinical data. All brain MR scans, pre-treatment and after 12 months of treatment were compared. In this follow up study, we did not perform MRI for our patients as it was not included for extension phase.

\section{Results}

\subsection{Safety}

The baseline characteristics of the patients are shown in Table 1. All of the patients experienced a mild headache and fever in the first 12 hours after injection, which in 4 of them was relieved after taking simple analgesics. In the other 2 , acute iatrogenic meningitis emerged. The CSF microbiologic analysis failed to show a pathogenic germ; nevertheless, the patients were treated with a two-week antibiotic regimen and recovered without any neurological sequelea. Apart from these, none of our patients complained about any unwanted outcome due to the MSC injection in the first month of therapy. We did not find any dramatic exacerbation or rapid progression of the disease after the injection. Out of 10,4 patients refused to co-operate further in the project without reporting any specific complication of MSC injection for their decision. We did not encounter any infection or neoplastic transformation in our patients after the five-year follow up. On the whole, all of our patients were relatively contented with the procedure and found it tolerable, although this treatment included rather aggressive procedures of bone marrow aspiration (BMA), and intrathecal injection. Even 2 of them requested to be injected with MSCs once more.

Table 1. Baseline characteristic and clinical data of the MS patients before and after autologous intratecal mesenchymal stem cell transplantation.

\begin{tabular}{|c|c|c|c|c|c|c|}
\hline & 1, Female & 2, Male & 3, Male & 4, Male & 5, Female & 6 , Female \\
\hline Age, $y$ & 39 & 28 & 28 & 39 & 22 & 35 \\
\hline Diagnosis (duration), y & $\operatorname{SPMS}^{\mathrm{a}}(14)$ & $\operatorname{SPMS}(7)$ & $\operatorname{SPMS}(7)$ & SPMS (12) & SPMS (3) & Devic's (16) NMO ${ }^{a}$ \\
\hline EDSS before injection & 6 & 5 & 5 & 5 & 3.5 & 5 \\
\hline Attacks after 5 years & 2 & None & None & None & 1 & 4 \\
\hline EDSS after 1 year & 6 & 2.5 & 5 & 6 & 4 & 5 \\
\hline EDSS after 5 years & 7 & 5 & 6.5 & 7 & 3 & 4 \\
\hline Complication & None & $\begin{array}{l}\text { Acute iatrogenic } \\
\text { meningits }\end{array}$ & None & None & None & $\begin{array}{l}\text { Acute iatrogenic } \\
\text { meningitis }\end{array}$ \\
\hline Further treatment ${ }^{b}$ & Azathioprine + Bethaferon & None & None & None & Mitoxantron & Azathioprine \\
\hline
\end{tabular}

a Abbreviations: EDSS, expanded disability status scale; NMO, neuromyelitis optica; SPMS, secondary progressive multiple sclerosis

$\mathrm{b}$ These treatments were initiated one year after the stem cell injection according to the patients' personal physicians' advice

\subsection{Efficacy}

The EDSS scores decreased in 1 patient, increased in 3, stabilized in 2 after the first one year. After five years the scores were decreased in 2 , unchanged in 1 , and increased in the others. It is interesting to mention that the EDSS score of one patient, who went through the iatrogenic meningitis, had a decline after one year (5 to 2.5) and after five years without any treatment had an EDSS score of 5. The other patient with meningitis was later diagnosed to have neuromyelitis optica. Her EDSS scores decreased 1 score after five years. However, she experiences 4 attacks after the injection in five years. Two patients had clinical attacks 6 months after the injection which were treated with intravenous methyl prednisolone. The patient with Devic's had an attack within the first months of the injection. Three of our patients received immunomodulatory treatment in the past four years, from whom 2 fairly showed no change in their EDSS score.

\section{Discussion}

There are different approaches regarding the stem cell therapy in MS (mesenchymal Vs. CNS-derived neural stem cell precursors and autologous vs. allogeneic) (6). The autologous MSCs are extracted through a BM aspiration of the patient and convey the least concerns upon graft rejection issues and need no pre- or post- transplant medications. These MSCs are believed to be attracted to the pathologic sites in the CNS and transform to cells that are capable of altering the microenvironment (19). Their major mechanism of action seems to be immunomodulation by secreting various cytokines and altering the balance of the T cells (20-22). Moreover, they can produce certain neurotrophic growth factors to assist the repair process through remyelination and neuroprotection $(3,4,6)$. Given that the other available treatments of MS mostly aim for controlling the pathologic immune response, the extra repairing ability of the MSCs is an additional asset. However, the conventional treatments 
regulate the immune system in a systemic nonspecific fashion. On the other hand, MSCs selectively limit CNS inflammation in the pathologic site, stimulate neurogenesis, protect axons, and promote remyelination (6, $23,24)$. However, some studies do not support the theory that the multi-potential MSCs actually differentiate into the much damaged neurons and glial cells in MS lesions. They merely perform their tasks through supporting the already existing back-up systems $(19,25)$. This may explain why we should expect less satisfying effects of MSC therapy when we use it in the later phases of MS, in which the major disability is due to rather irreversible neuroaxonal loss. To the best of our knowledge, our study is the first report of patients with MS who were treated with intrathecal MSCs and followed for five years. The safety and efficacy of MSCs therapy in MS have been investigated in a limited number of studies following our first report (16). Yamout et al. explored the safety and therapeutic effect of intrathecal MSCs in ten patients with advanced MS and reported some clinical but not radiological improvement after 6 months. They concluded that this method is safe and tolerable for the patients, and they did not face any severe adverse event (17). In a phase $1 / 2$ pilot study, Karussis et al. showed safety and feasibility of combined intrathecal and intravenous injection of MSCs in patients with MS. Among 15 patients, the EDSS score remained unchanged in 4 patients, and was reduced by 0.5 degree in 5 patients. It improved by 1.0 degree in 1 patient, by 1.5 degrees in 3 patients, by 2.0 degrees in 1 patient, and by 2.5 degrees in 1 patient. The EDSS score did not deteriorate in any of the patients (15). In our study, it also seemed that those who had EDSS scores lower than 5, had a better outcome (either stabilization or improvement). Other studies have reported an acceptable efficacy for MSC therapy in MS, even though they used different methods. As expected, they also suggested that the clinical response strongly relates to the phase of the disease course in which the therapy is carried out, whereas rapidly evolving MS with relapses and remissions are the cases that can take more advantage (26). Our study had very small patient numbers to conclude the efficacy of this treatment, and the extended follow up was mostly focused on reporting any long-term complications. Nonetheless, we can announce that some of our patients experienced a beneficial effect with MSC therapy (either stabilization or improvement), and none revealed a dramatic adverse response (indicating no effect of the MSC therapy), and we also found that the autologous intrathecal MSC injection is harmless even after a five-year follow up. It seemed that those of our patients who received an additional immunomodulatory treatment one year after the injection, ended up in a better clinical status. Considering that our patients had just one session of MSC injection in five years; one possible theory is that therapeutic effects of the injected MSCs diminishes overtime, which is less likely, because the transformed MSCs were found in the autopsies at least two years after injection (27). Another theory is that the MSCs need reinforcements over time which can be provided by an additional immunomodulatory medication or probably by redoing the MSC therapy. Of course these questions remain to be answered in the upcoming larger scale trials. We had an interesting patient with Devic's neuromyelitis optica that was treated with MSC. Her clinical status decreased one score after five years of injection, nevertheless few relapses kept occurring even with azathioprine treatment. All the same, given the degenerative nature of this disease, it seems that she may have actually benefited from the MSC injection. However, multiple factors could have influenced the course of this disease, which makes it difficult to have a precise deduction.

Intrathecal MSC therapy can be considered as a safe and partially effective treatment in patients with secondary progressive MS. However, future controlled studies with larger sample sizes and long-term follow up with both clinical and MRI features of the patients are needed to answer lists of questions about the efficacy of this type of cell therapy.

\section{Acknowledgements}

We would like to thank the research development office of Sina Hospital for their assistance in preparing the manuscript.

\section{Authors' Contribution}

Gathering the data/review the manuscript: Mohammad Ali Sahraian; preparing the stem cells/review the manuscript: Mandana Mohyeddin Bonab; Preparing the final results/writing the manuscript: Sanaz Ahmadi Karvigh; Injecting the stem cells/review the manuscript: Sepideh Yazdanbakhsh; Preparing the stem cells/ review the manuscript: Behrouz Nikbin; Selecting and examining the patients/review the manuscript: Jamshid Lotfi.

\section{Financial Disclosure}

There is not any financial disclosure.

\section{Funding/Support}

The research was supported by Tehran University of Medical Sciences.

\section{References}

1. Kantarci $\mathrm{OH}$. Genetics and natural history of multiple sclerosis. Semin Neurol. 2008;28(1):7-16.

2. Kremenchutzky M, Rice GP, Baskerville J, Wingerchuk DM, Ebers GC. The natural history of multiple sclerosis: a geographically based study 9: observations on the progressive phase of the disease. Brain. 2006;129(Pt 3):584-94.

3. Petratos S, Azari MF, Ozturk E, Papadopoulos R, Bernard CC. Novel therapeutic targets for axonal degeneration in multiple sclerosis. J Neuropathol Exp Neurol. 2010;69(4):323-34.

4. Martino G, Franklin RJ, Baron Van Evercooren A, Kerr DA. Stem 
cell transplantation in multiple sclerosis: current status and future prospects. Nat Rev Neurol. 2010;6(5):247-55.

5. Crigler L, Robey RC, Asawachaicharn A, Gaupp D, Phinney DG. Human mesenchymal stem cell subpopulations express a variety of neuro-regulatory molecules and promote neuronal cell survival and neuritogenesis. Exp Neurol. 2006;198(1):54-64.

6. Siatskas C, Payne NL, Short MA, Bernard CC. A consensus statement addressing mesenchymal stem cell transplantation for multiple sclerosis: it's time! Stem Cell Rev. 2010;6(4):500-6.

7. Tirotta E, Carbajal KS, Schaumburg CS, Whitman L, Lane TE. Cell replacement therapies to promote remyelination in a viral model of demyelination. J Neuroimmunol. 2010;224(1-2):101-7.

8. Pittenger MF, Mackay AM, Beck SC, Jaiswal RK, Douglas R, Mosca JD, et al. Multilineage potential of adult human mesenchymal stem cells. Science.1999;284(5411):143-7.

9. Yang J, Rostami A, Zhang GX. Cellular remyelinating therapy in multiple sclerosis. J Neurol Sci. 2009;276(1-2):1-5.

10. Bai L, Lennon DP, Eaton V, Maier K, Caplan AI, Miller SD, et al. Human bone marrow-derived mesenchymal stem cells induce Th2polarized immune response and promote endogenous repair in animal models of multiple sclerosis. Glia. 2009;57(11):1192-203.

11. Gordon D, Pavlovska G, Glover CP, Uney JB, Wraith D, Scolding NJ. Human mesenchymal stem cells abrogate experimental allergic encephalomyelitis after intraperitoneal injection, and with sparse CNS infiltration. Neurosci Lett. 2008;448(1):71-3.

12. Kassis I, Grigoriadis N, Gowda-Kurkalli B, Mizrachi-Kol R, Ben-Hur T, Slavin S, et al. Neuroprotection and immunomodulation with mesenchymal stem cells in chronic experimental autoimmune encephalomyelitis. Arch Neurol. 2008;65(6):753-61.

13. Zappia E, Casazza S, Pedemonte E, Benvenuto F, Bonanni I, Gerdoni E, et al. Mesenchymal stem cells ameliorate experimental autoimmune encephalomyelitis inducing T-cell anergy. Blood. 2005;106(5):1755-61.

14. Zhang J, Li Y, Chen J, Cui Y, Lu M, Elias SB, et al. Human bone marrow stromal cell treatment improves neurological functional recovery in EAE mice. Exp Neurol. 2005;195(1):16-26.

15. Karussis D, Karageorgiou C, Vaknin-Dembinsky A, Gowda-Kurkalli B, Gomori JM, Kassis I, et al. Safety and immunological effects of mesenchymal stem cell transplantation in patients with multiple sclerosis and amyotrophic lateral sclerosis. Arch Neurol.
2010;67(10):1187-94

16. Mohyeddin Bonab M, Yazdanbakhsh S, Lotfi J, Alimoghaddom K, Talebian F, Hooshmand F, et al. Does mesenchymal stem cell therapy help multiple sclerosis patients? Report of a pilot study. Iran J Immunol. 2007;4(1):50-7.

17. Yamout B, Hourani R, Salti H, Barada W, El-Hajj T, Al-Kutoubi A, et al. Bone marrow mesenchymal stem cell transplantation in patients with multiple sclerosis: a pilot study. J Neuroimmunol. 2010;227(1-2):185-9.

18. Freedman MS, Bar-Or A, Atkins HL, Karussis D, Frassoni F, Lazarus $\mathrm{H}$, et al. The therapeutic potential of mesenchymal stem cell transplantation as a treatment for multiple sclerosis: consensus report of the International MSCT Study Group. Mult Scler 2010;16(4):503-10.

19. Marmont AM. New horizons in the treatment of autoimmune diseases: immunoablation and stem cell transplantation. Annu Rev Med. 2000;51:115-34.

20. Beyth S, Borovsky Z, Mevorach D, Liebergall M, Gazit Z, Aslan H, et al. Human mesenchymal stem cells alter antigen-presenting cell maturation and induce T-cell unresponsiveness. Blood. 2005;105(5):2214-9.

21. Lassmann H. Stem cell and progenitor cell transplantation in multiple sclerosis: the discrepancy between neurobiological attraction and clinical feasibility. J Neurol Sci. 2005;233(1-2):83-6.

22. Le Blanc K, Tammik C, Rosendahl K, Zetterberg E, Ringden O. HLA expression and immunologic properties of differentiated and undifferentiated mesenchymal stem cells. Exp Hematol. 2003;31(10):890-6.

23. Gregory CA, Prockop DJ, Spees JL. Non-hematopoietic bone marrow stem cells: molecular control of expansion and differentiation. Exp Cell Res. 2005;306(2):330-5.

24. Krampera M, Pasini A, Pizzolo G, Cosmi L, Romagnani S, Annunziato F. Regenerative and immunomodulatory potential of mesenchymal stem cells. Curr Opin Pharmacol. 2006;6(4):435-41.

25. Caplan AI, Dennis JE. Mesenchymal stem cells as trophic mediators. J Cell Biochem. 2006;98(5):1076-84.

26. Uccelli A, Mancardi G. Stem cell transplantation in multiple sclerosis. Curr Opin Neurol. 2010;23(3):218-25.

27. Garzon-Muvdi T, Quinones-Hinojosa A. Neural stem cell niches and homing: recruitment and integration into functional tissues. ILAR J. 2009;51(1):3-23. 